\title{
Effects of irradiation and leukoreduction on down-regulation of CXCL-8 and storage lesion in stored canine whole blood
}

\author{
Hayoung Yang ${ }^{1}$, Woosun $\mathrm{Kim}^{1}$, Junwoo Bae ${ }^{1}$, Hyunwoo Kim ${ }^{1}$, Sangki Kim ${ }^{2}$, Jihye Choi ${ }^{1}$, Jinho Park ${ }^{3}$, Dong-In Jung ${ }^{4}$, \\ HongBum $\mathrm{Koh}^{1, *}$, DoHyeon $\mathrm{Yu}^{4, *}$ \\ ${ }^{1}$ College of Veterinary Medicine, Chonnam National University, Gwangju 61186, Korea \\ ${ }^{2}$ College of Industrial Science, Kongju National University, Yesan 32439, Korea \\ ${ }^{3}$ College of Veterinary Medicine, Chonbuk National University, Iksan 54596, Korea \\ ${ }^{4}$ Institute of Animal Medicine, College of Veterinary Medicine, Gyeongsang National University, Jinju 52828, Korea
}

\begin{abstract}
White blood cells (WBCs) and storage period are the main factors of transfusion reactions. In the present study, cytokine/chemokine concentrations after leukoreduction (LR) and irradiation (IR) in stored canine whole blood were measured. Red blood cell storage lesion caused by IR and LR were also compared. Blood samples from 10 healthy Beagles were divided into four groups (no treatment, LR-, IR-, and LR + IR-treated). Leukocytes were removed by filtration in the LR group and gamma radiation (25 Gy) was applied in the IR group. Immunologic factors (WBCs, interleukin-6 [IL-6], C-X-C motif chemokine ligand 8 [CXCL-8], and tumor necrosis factor-alpha) and storage lesion factors (blood $\mathrm{pH}$, potassium, and hemolysis) were evaluated on storage days $0,7,14,21$, and 28 . Compared to the treated groups, IL-6 and CXCL-8 concentrations during storage were significantly higher in the control (no treatment) group. LR did not show changes in cytokine/chemokine concentrations, and storage lesion presence was relatively mild. IR significantly increased CXCL-8 after 14 days of storage, but IR of leukoreduced blood did not increase CXCL-8 during 28 days of storage. Storage lesions such as hemolysis, increased potassium, and low $\mathrm{pH}$ were observed 7 days after IR and storage of blood, regardless of LR. IR of leukoreduced blood is beneficial to avoid immune reactions; however, storage lesions should be considered upon storage.
\end{abstract}

Keywords: Blood preservation; Irradiation; Leukocyte reduction procedures; Transfusion; Cytokine

\section{Introduction}

Blood transfusions are commonly used in small animal practice, but they have potential adverse effects such as febrile nonhemolytic transfusion reaction (FNHTR), transfusionassociated graft versus host disease (TA-GvHD), immunologic or non-immunologic hemolysis, transfusion-related acute lung injury (TRALI), transfusion-associated circulatory overload, and storage lesion (i.e., biochemical and biomechanical changes depending on red blood cell [RBC] storage time) $[1,4,14,23]$. To prevent such transfusion reactions, compatibility testing, donor selection, limitation storage, leukoreduction (LR), and additive solutions are used in human medicine [17,26,28].
White blood cells (WBCs) are thought to be the main cause of FNHTR, resulting from the production of a cytokine that mediates inflammatory disease [5,37]. In humans, TA-GvHD is a WBC-related transfusion reaction and is a fatal disease that causes the lymphocytes involved in blood transfusions to grow in the body of the host, often an immunosuppressed patient [7,32].

Presence of a storage lesion, a non-immunologic transfusion reaction, implies the occurrence of biochemical and biomechanical changes that are dependent on the storage time of RBCs. During RBC storage, 2, 3-diphosphoglycerate is insufficient to prevent oxygen transfer from hemoglobin and, ultimately, delivery of oxygen to tissues. Moreover, free hemoglobin and 
microparticle levels are increased, which might have harmful effects, such as TRALI and thrombosis, on recipients $[12,15,20]$. Numerous mechanisms cause biochemical and biomechanical changes, and WBCs remaining in RBC bags can contribute to the development of storage lesions [25,26]. Despite the adverse effects of storage lesions, the practice at many veterinary hospitals is to use the oldest blood in their inventory [16,18].

There are several options to prevent these transfusion reactions. One is LR, which removes leukocytes and platelets from blood products by filtration. It has been reported that LR can reduce the probability of occurrence of FNHTR, transfusion-related immunomodulation, and transmission of specific viruses by removing leukocytes in human products $[4,37]$. LR in dogs is reported to reduce inflammatory response by preventing the accumulation of cytokines derived from WBCs [9,24]. In human hospitals, TA-GvHD prevention involves the irradiation (IR) of blood to inactivate lymphocytes. The number and viability of lymphocytes that are incompletely eliminated through filters are related to the risk of disease occurrence. However, there is limited information about the IR of blood in veterinary medicine.

The presence of residual WBCs in stored blood and the storage period have potentially deleterious effects on blood products. Therefore, the objective of this study was to measure cytokine/chemokine concentrations in canine whole blood during various storage periods following IR and/or LR treatments. The levels of various RBC storage lesions resulting from the IR and LR treatments were compared. The hypothesis was that the reduction of cytokines and chemokines from nucleated cells in irradiated blood was similar to that in leukoreduced blood and that IR of canine whole blood is suitable as a pre-storage treatment.

\section{Materials and Methods}

\section{Dogs}

Blood samples used in the study were obtained from 10 healthy female Beagle dogs ranging in age from 1 to 3 years and in weight from 11 to $15 \mathrm{~kg}$. The dogs were healthy, based on clinical examinations performed by veterinarians and the results of complete blood cell counts. Food and water were provided ad libitum. The dogs were fasted for one day before blood collection to minimize lipemia-induced hemolysis (in vitro). This study was approved by Chonnam National University Animal Care and Use Committee (approval No. CNU IACUC-YB-R-2017-06).

\section{Collection of blood}

Blood was collected using a standard protocol. Each dog was positioned in lateral recumbency for blood collection. The jugular venipuncture site was clipped and prepared with an aseptic technique to prevent bacterial contamination. Intradermal injection of a lidocaine and bicarbonate mixture (10:1) was used for local anesthesia. Each dog was sedated with intravenous alfaxalone $(3 \mathrm{mg} / \mathrm{kg})$. Blood was collected $(200 \mathrm{~mL})$ by gravity assistance in a citrate-phosphate-dextrose (CPD)-containing bag following venipuncture of the jugular vein using a $16 \mathrm{G}$ needle. As soon as blood collection was complete, each dog was intravenously administered lactated Ringer's solution to replace the lost volume and was monitored for complications. The filled CPD bags were divided into control group $(100 \mathrm{~mL})$ and filtered blood $(100 \mathrm{~mL})$ groups. Blood was collected by using a closed collection system and leukocytes were removed by filtration (Blood Bag with RBC Filter and Safety DDA Plus 3 Quadruple $400 \mathrm{~mL}$; Tae-Chang Industrial, Korea). This blood bag contains $56 \mathrm{~mL}$ of anticoagulant for a $400 \mathrm{~mL}$ blood collection, and half of the anticoagulant was discarded via a closed system prior to collecting $200 \mathrm{~mL}$ of whole blood. Three weeks later, the procedure was repeated after packed cell volume recovery. The two blood bags from each dog were gamma irradiated using a 25 Gy dose (Gammacell 3000 Elan; Best Theratronics, Canada). All blood bags from untreated control and LR-, IR-, LR + IR-treated dogs were maintained at $4^{\circ} \mathrm{C}$ in a refrigerator and mixed gently at least once a day.

\section{Sample analysis}

Aliquots $(10 \mathrm{~mL})$ of blood were aseptically obtained from all blood bags on storage days $0,7,14,21$, and 28 . A portion of the aliquot was immediately analyzed using an automatic hematology analyzer (Procyte Dx; IDEXX Laboratories, USA) and a blood gas analyzer (Nova pHOX Analyzer; Nova Biomedical, USA). The WBC count, $\mathrm{pH}$, and potassium level data were collected from the analyzer output. Hemolysis was determined by visual observation. The remaining aliquot portions were immediately centrifuged at $1,850 \times \mathrm{g}$ for $15 \mathrm{~min}$ at $4^{\circ} \mathrm{C}$ to harvest plasma, which was divided into six microcentrifuge tubes and stored at $-80^{\circ} \mathrm{C}$ until cytokine/chemokine analyses.

\section{Cytokine and chemokine assessment}

Canine interleukin-6 (IL-6), C-X-C motif chemokine ligand 8 (CXCL-8), and tumor necrosis factor-alpha (TNF- $\alpha$ ) were measured by using sandwich enzyme-linked immunosorbent assay (ELISA) based on commercially available matched canine antibody pairs (Duoset ELISA Development Kit; R\&D Systems, USA). Assays were performed in duplicate according to the manufacturer's instructions. Briefly, wells of a 96-well plate were coated overnight with capture antibody (goat anti-canine target antibodies). Then, the plate was blocked with $1 \%$ bovine serum albumin in phosphate buffered saline and washed with $0.05 \%$ Tween 20 . After washing, detection antibody (biotinylated goat anti-canine target antibody) was added, and detection was achieved through the addition of streptavidinhorseradish peroxidase and tetramethylbenzidine liquid 
substrate solution. The optical density of each well was measured using a microplate reader (Versamax ELISA Microplate Reader; Molecular Devices, USA) and analyzing software (Softmax Pro; Molecular Devices). Plasma samples from all dogs were used as the baseline for the spike and recovery experiments. The spiking levels were determined from the assay detection limits. Recoveries of $75 \%$ to $125 \%$ were deemed acceptable. The lower limits of detection for IL-6, TNF- $\alpha$, and CXCL- 8 were $62.5 \mathrm{pg} / \mathrm{mL}, 15.6 \mathrm{pg} / \mathrm{mL}$, and 15.6 $\mathrm{pg} / \mathrm{mL}$, respectively.

\section{Statistical analyses}

Statistical analyses were performed using the IBM SPSS for Windows (ver. 23; IBM, USA). All results were explored and tested for data normality by the Shapiro-Wilk test. Cytokine/ chemokine concentrations, potassium level, and $\mathrm{pH}$ were compared by two-way repeated ANOVA with evaluations for group and storage time effects. For post hoc analysis, a Tukey test was used. The significance level for all statistical tests was predetermined at $p<0.05$.

\section{Results}

\section{Cytokine and chemokine changes}

IL-6 concentrations did not change with time in all groups. However, the IL-6 concentration of the control group was greater than that of the other groups at each storage time. IL-6 concentrations were significantly different between the control and LR + IR groups $(p<0.05)$. The results indicated that the IL-6 concentrations of LR-, IR-, and LR + IR-treated groups tended to be lower than that of the control group (Fig. 1). The

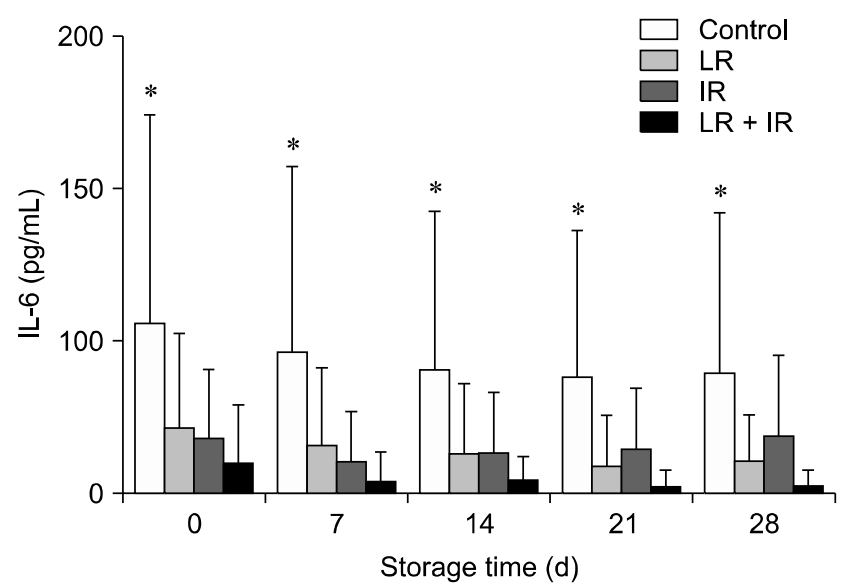

Fig. 1. Variations in mean interleukin-6 (IL-6) concentrations (n $=10$ ) during storage. Data are presented as mean \pm SD. Note that the minimum scale of the $y$-axis is the detection limit of the assay $(62.5 \mathrm{pg} / \mathrm{mL})$. LR, leukoreduction; IR, irradiation. *Significant difference between control and LR + IR at each storage time $(p<0.05)$.
CXCL-8 concentration of the control group increased after 21 days of storage. CXCL-8 concentrations in the LR group were less than the detectable limit at all storage times. However, the CXCL-8 concentration of the IR group was markedly increased over that of the other groups after 7 days of storage.

With increasing storage time, the CXCL-8 concentrations of the LR + IR group increased, but the levels were consistently lower than that of the control group (Fig. 2). TNF- $\alpha$ concentrations were less than the detectable limit in all groups and at all storage times (data not shown).

\section{Storage lesion presence}

The assessed storage lesion types included presence of hemolysis as well as potassium level and $\mathrm{pH}$. Hemolysis was not visually evident in any group until storage day 7 , but was visually observed after 14 days in the IR and LR + IR groups (Fig. 3). The mean potassium value of the control group increased with storage time, whereas that of the LR group displayed temporal stability. The mean potassium values of the IR and LR + IR groups sharply increased within the initial 7 days following IR, and the IR group displayed a marked increase compared to the other groups (Fig. 4).

The $\mathrm{pH}$ of all groups decreased in a time-dependent manner. The LR group displayed a relatively stable rate of change compared to those in the other groups. After 7 days of storage, the $\mathrm{pH}$ in the IR group had markedly decreased to $<6.5$, and the $\mathrm{pH}$ of the IR group was less than the detectable limit ( $\mathrm{pH}$ 6.5) after 7 days. The $\mathrm{pH}$ of the control and LR + IR groups was less than the detectable limit (pH 6.5) after 21 days of storage (Fig. 5).

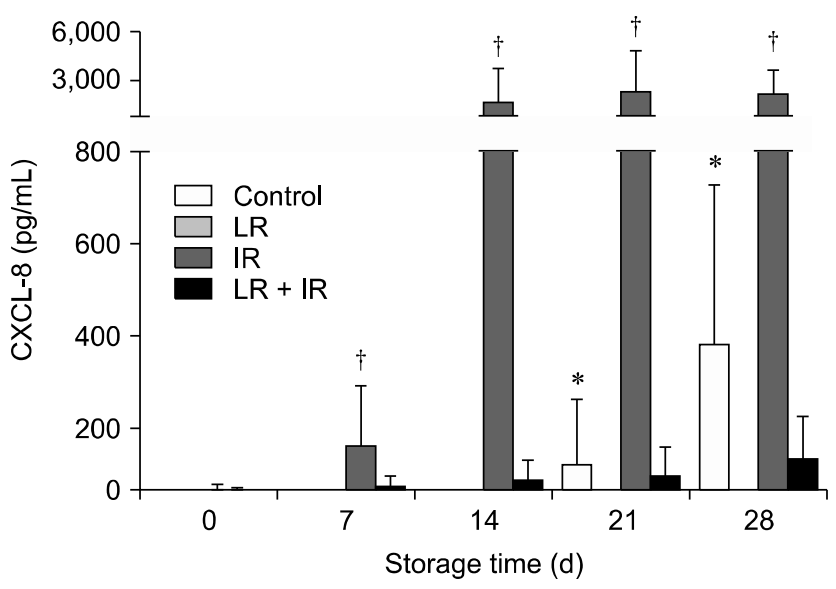

Fig. 2. Variations in mean $\mathrm{C}-\mathrm{X}-\mathrm{C}$ motif chemokine ligand 8 (CXCL-8) concentrations $(n=10)$ during storage. Data are presented as mean \pm SD. The $y$-axis of the mean concentration is divided into two sections. Note that the minimum scale of the $y$-axis is the detection limit of the assay $(15.6 \mathrm{pg} / \mathrm{mL}) . \quad L R$, leukoreduction; $I R$, irradiation. *Significant difference between control and LR at indicated storage times $(p<0.05)$; ${ }^{\dagger}$ Significant difference between IR and control at each storage time $(p<0.05)$. 


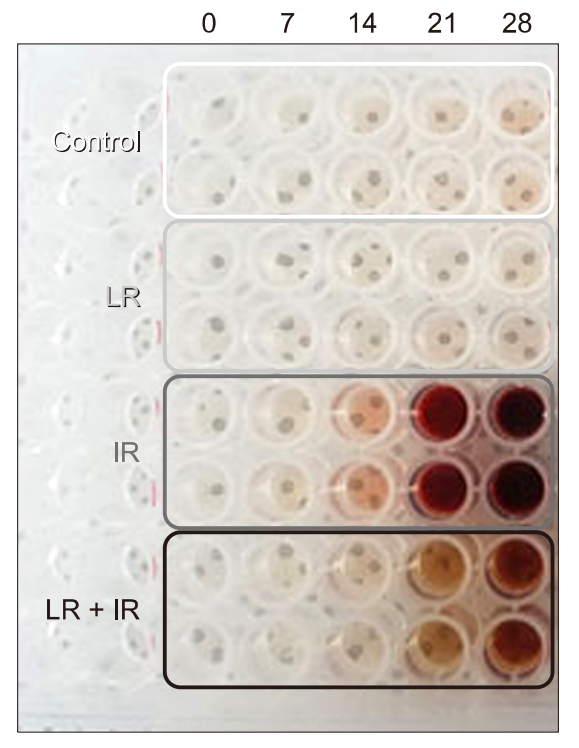

Fig. 3. Visual observation of hemolysis during storage. The samples bounded by the blue, green, yellow, and purple border are the control samples, leukoreduced (LR) samples, irradiated (IR) samples, and leukoreduced and irradiated (LR + IR) samples, respectively. The numbers represent the days of storage.

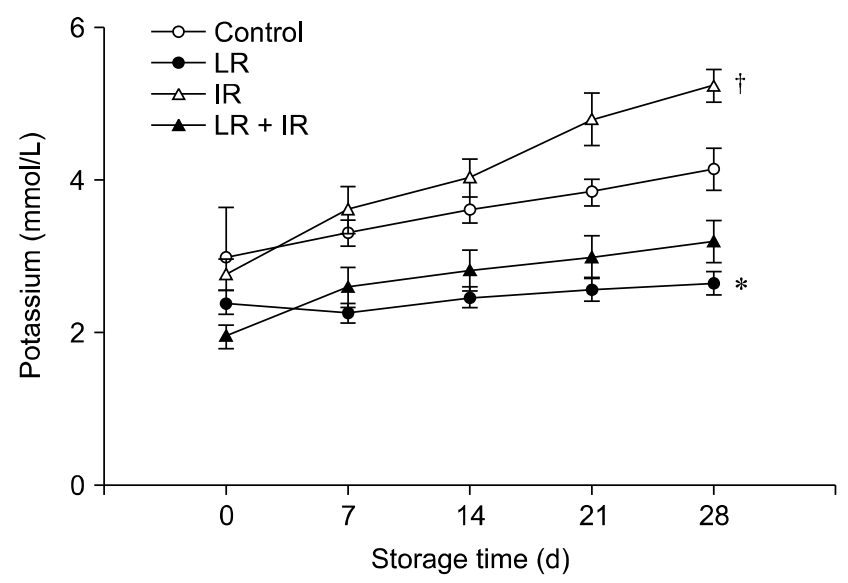

Fig. 4. Variations in mean potassium values $(n=10)$ during storage. Data are presented as mean $\pm \mathrm{SD}$. LR, leukoreduction; IR, irradiation. ${ }^{*}$ LR group was significantly different from the other groups $(p<0.05)$; ${ }^{\dagger}$ IR group was significantly different from the other groups $(p<0.05)$.

\section{Discussion}

Cytokines have a key role in the development of the inflammatory response. IL-6, CXCL- 8 , and TNF- $\alpha$ contribute to the inflammatory response $[9,36]$ and are significant mediators of the proinflammatory phase, leading to an elevation of body temperature. In addition, these cytokines can stimulate other inflammatory cascades. In the present study, these three

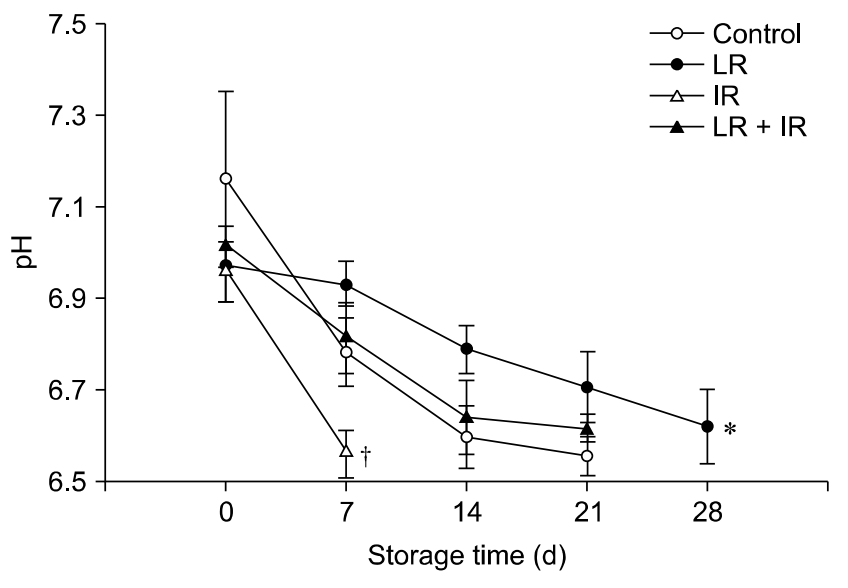

Fig. 5. Variations in mean $\mathrm{pH}(\mathrm{n}=10)$ during storage. Data are presented as mean $\pm \mathrm{SD}$. The $\mathrm{pH}$ of the IR group was less than the detectable limit ( $\mathrm{pH}$ 6.5) after 7 days. The $\mathrm{pH}$ values of the control and $L R+I R$ groups were less than the detectable limit (pH 6.5) after 21 days. LR, leukoreduction; IR, irradiation. * $2 R$ group was significantly different from the other groups $(p<$ $0.05) ;{ }^{\dagger} I R$ group was significantly different from the other groups $(p<0.05)$.

indicator cytokines were analyzed in vitro to emulate the inflammatory reactions in vivo and the blood transfusion reactions. The concentrations of IL- 6 , TNF- $\alpha$, and CXCL- 8 were analyzed in four groups (control, LR, IR, and LR + IR) for a variety of storage times. The IL- 6 concentration in the control group was significantly higher than that in the other groups at all storage times. The CXCL- 8 concentration in the IR and control groups increased with storage time, while TNF- $\alpha$ concentrations were undetectable in all groups.

Human studies have shown that leukocytes partially generate cytokines in the stored blood. IL-1, IL-6, CXCL-8, TNF- $\alpha$, and regulated upon activation normal T-cell expressed and secreted (RANTES) are significantly increased in stored blood [33]. These proinflammatory cytokines have pyrogenic activity and are related to FNHTR [14,28,29]. FNHTR is the most common complications in human and dogs [3,23]. A human tertiary care hospital recently conducted a retrospective analysis comparing the rate of FNHTR following LR and non-LR transfusion. The incidence of FNHTR displayed an approximately 5-times difference between LR and non-LR patients; other studies have similarly reported at least a 2- to 20-times difference between the incidence rates of FNHTR in LR and non-LR transfusions $[2,4,22,28]$. However, there have been few published descriptions of the incidence rate of FNHTR between LR and non-LR transfusions in veterinary medicine. In a study of $210 \mathrm{dogs}$ that received packed RBCs, nearly half of the blood-transfused dogs developed fever [23]. This implies there is a need for the application of LR blood to reduce the risk of developing FNHTR. In this study, there was no rise in the CXCL-8 level in 
the LR group, and groups in which leucocytes were not removed showed a time-dependent increase in CXCL-8 level. This tendency was similar to the results in another study [9]. Based on this previous study, CXCL-8 derived from leukocytes is believed to be a contributor to FNHTR. The present results suggest that CXCL-8 eliminated through LR filtration may be related to the low incidence of FNHTR in dogs. This relationship is of great benefit to patients with an underlying disease. Patients who receive transfused blood may suffer from the most critical diseases, and the chance of a poor outcome due to even a small side effect is likely [13].

IL-6 is a type of pyrogen that has a role in mediating the acute phase response and fever. In this study, the IL- 6 concentration in the control group was higher than that in the other groups at every storage time, but it did not increase in a time-dependent manner. In a previous veterinary study, there was a trend toward higher concentrations of IL- $1 \beta$ and TNF- $\alpha$, but no timedependent increase was observed [8,24,27]. While the cytokines were different, the upward trend was similarly observed in the control group.

The LR filters used in the experiment removed more than $99.9 \%$ of the leukocytes through adsorption. The mechanism of LR filtration is charge-based adhesion of negatively-charged leukocytes and platelets to the filter material by van der Waals and electrostatic forces $[6,10]$. Considering the results of the experiments and those in a series of other studies, it is reasonable to suggest that cytokines can be partially absorbed by a filter. As a result, the reduction in cytokine levels will have a positive effect on reducing transfusion reactions. Further research needs to be done to devise filters that can reduce the concentration of some cytokines in veterinary medicine.

Approximately ten years ago, LR blood began to be widely used in human hospitals. However, it has not been widely used in veterinary medicine. According to a recent study, only two of 53 private referral hospitals and veterinary teaching hospitals use LR blood [16,18,21]. The reason is that the additional expense of the LR filter does not outweigh the benefits. However, in one study, transfusions were performed in 210 cases and 84 transfusion-related complications followed [23]. There are also studies documenting that longer storage blood transfusion times may negatively affect the outcome in dogs [13]. As a solution, many studies have suggested that the use of LR can prevent the storage lesion occurrence $[9,11,15,24]$. In this study, the cytokine concentration following LR was not increased, and hemolysis and $\mathrm{pH}$ change remained relatively stable throughout the storage period. Despite these LR-related benefits, most veterinary hospitals still practice a 'first-in, first-out' blood storage strategy that has an economic advantage [18]. It is suggested that LR can be a solution to the conflict between economic benefits and transfusion reactions.

TA-GvHD is a fatal complication that occurs mostly in immunosuppressed patients who are transfused with lymphocyte- containing blood components. The transplanted lymphocytes can spread to skin, gut, and liver and can cause various problems; symptoms of this disease include fever, skin rash, diarrhea, hepatitis, and pancytopenia caused by bone marrow hypoplasia, and TA-GvHD mortality can exceed $90 \%$. Currently, the best way to prevent TA-GvHD is IR of blood to inactivate the remaining lymphocytes $[1,31,35]$. TA-GvHD has only been reported as an experimental model in dogs. To the author's best knowledge, this study is the first to report the cytokine/chemokine concentrations, potassium concentrations, and $\mathrm{pH}$ levels in canine irradiated blood. Our hypothesis is that irradiated blood has the same effect as leukoreduced blood in terms of reducing cytokine/chemokine concentrations in nucleated cells. This is because gamma rays blunt the activation of the nucleated cell. Regardless, research on irradiated blood is required in veterinary medicine. In particular, research on TA-GvHD in dogs that are treated with immunosuppression therapy (e.g., purine analogs) is anticipated.

In the present study, the CXCL-8 concentration increased sharply in the irradiated blood group. In addition, rapid changes in potassium and $\mathrm{pH}$ were observed after gamma IR. Furthermore, the hemolysis level increased after 14 storage days in the IR group. Human studies have shown that gamma IR of RBCs intensifies the leakage of potassium and increases the level of extracellular potassium [19,34]. Furthermore, a low $\mathrm{pH}$ accelerates hyperkalemia because of the shift of potassium from the intracellular to the extracellular compartments. Potassium levels are clinically important in massive transfusions. Another study retrospectively investigated the association of transfusion with hyperkalemia in humans and observed that the hyperkalemia could induce cardiac arrest [30]. The increase in free hemoglobin resulting from hemolysis decreases the ability of oxygen delivery during transfusion. This type of storage lesion occurs in older blood. The increase in free hemoglobin stimulates endothelium function, consumes platelets, and promotes clotting factors that cause thromboembolic disease and coagulation failure [12]. Human-based research has suggested that the irradiated blood should be used within 14 days [31]. IR is unsuitable for reducing storage lesion occurrence, as shown by the results in this study. However, IR of leukoreduced blood produced a rather mild level of storage lesions compared to irradiated-only blood. In humans, IR of blood is indicated, while, in dogs, IR of leukoreduced blood immediately preceding a transfusion should be considered.

There are several potential limitations in this study. One is that the blood samples were not analyzed for free hemoglobin. Although hemolysis was confirmed visually, there were no quantitative comparisons via the examination of free hemoglobin. Additionally, it is difficult to distinguish whether potassium had leaked or not leaked in the irradiated plasma. A second limitation is that the data were solely from experiments done in vitro. Even though cytokine/chemokine increases can mediate 
inflammation in vitro, the relationship between the quantities of cytokine/chemokine in transfusion and inflammation in vivo is unclear. Therefore, additional research in vivo is needed based on the obtained in vitro data. Another limitation is that the appropriate gamma IR dose is unknown for dogs. However, the American Association of Blood Banks and the Japanese Society of Blood Transfusions guidelines recommend a dose of $25 \mathrm{~Gy}$ to the central area of the blood component. A minimum dose of $25 \mathrm{~Gy}$, but not to exceed $50 \mathrm{~Gy}$, was recommended by the British Committee for Standards in Haematology Transfusion Task Force [31]. Functional evaluation of lymphocytes after IR should be undertaken in further canine studies.

\section{Acknowledgments}

This research was supported by the Basic Science Research Program through the National Research Foundation of Korea (NRF) funded by the Ministry of Science, ICT \& Future Planning (NRF-2017R1D1A1B03034904) and Cooperative Research Program for Agriculture Science and Technology Development (PJ01284305), Rural Development Administration.

\section{Conflict of Interest}

The authors declare no conflicts of interest.

\section{References}

1. Aoun E, Shamseddine A, Chehal A, Obeid M, Taher A. Transfusion-associated GVHD: 10 years' experience at the American University of Beirut-Medical Center. Transfusion 2003, 43, 1672-1676.

2. Bhattacharya P, Marwaha N, Dhawan HK, Roy P, Sharma RR. Transfusion-related adverse events at the tertiary care center in North India: an institutional hemovigilance effort. Asian J Transfus Sci 2011, 5, 164-170.

3. Bilgin YM, van de Watering LM, Brand A. Clinical effects of leucoreduction of blood transfusions. Neth J Med 2011, 69, 441-450.

4. Blajchman MA. Immunomodulation and blood transfusion. Am J Ther 2002, 9, 389-395.

5. Blumberg N, Gettings KF, Tumer C, Heal JM, Phipps RP. An association of soluble CD40 ligand (CD154) with adverse reactions to platelet transfusions. Transfusion 2006, 46, 1813-1821.

6. Bruil A, Beugeling T, Feijen J, van Aken WG. The mechanisms of leukocyte removal by filtration. Transfus Med Rev 1995, 9, 145-166.

7. Burns LJ, Westberg MW, Burns CP, Klassen LW, Goeken NE, Ray TL, Macfarlane DE. Acute graft-versus-host disease resulting from normal donor blood transfusions. Acta Haematol 1984, 71, 270-276.

8. Callan MB, Patel RT, Rux AH, Bandyopadhyay S, Sireci AN, O'Donnell PA, Ruane T, Sikora T, Marryott K, Sachais
BS, Hod EA. Transfusion of 28-day-old leucoreduced or non-leucoreduced stored red blood cells induces an inflammatory response in healthy dogs. Vox Sang 2013, 105, 319-327.

9. Corsi R, McMichael MA, Smith SA, O'Brien M, Herring J, Ngwenyama TR, Galligan A, Beloshapka AN, Deng P, Swanson KS. Cytokine concentration in stored canine erythrocyte concentrates. J Vet Emerg Crit Care (San Antonio) 2014, 24, 259-263.

10. Dzik S. Mechanisms of leukocyte removal by filtration. Curr Stud Hematol Blood Transfus 1994, 60, 123-133.

11. Graf C, Raila J, Schweigert FJ, Kohn B. Effect of leukoreduction treatment on vascular endothelial growth factor concentration in stored canine blood transfusion products. Am J Vet Res 2012, 73, 2001-2006.

12. Grimshaw K, Sahler J, Spinelli SL, Phipps RP, Blumberg N. New frontiers in transfusion biology: identification and significance of mediators of morbidity and mortality in stored red blood cells. Transfusion 2011, 51, 874-880.

13. Hann L, Brown DC, King LG, Callan MB. Effect of duration of packed red blood cell storage on morbidity and mortality in dogs after transfusion: 3,095 cases (2001-2010). J Vet Intern Med 2014, 28, 1830-1837.

14. Heddle NM. Pathophysiology of febrile nonhemolytic transfusion reactions. Curr Opin Hematol 1999, 6, 420-426.

15. Herring JM, Smith SA, McMichael MA, O'Brien M, Ngwenyama TR, Corsi R, Galligan A, Beloshapka AN, Deng P, Swanson KS. Microparticles in stored canine RBC concentrates. Vet Clin Pathol 2013, 42, 163-169.

16. Holowaychuk MK, Musulin SE. The effect of blood usage protocol on the age of packed red blood cell transfusions administered at 2 veterinary teaching hospitals. J Vet Emerg Crit Care (San Antonio) 2015, 25, 679-683.

17. Jackman RP, Deng X, Bolgiano D, Utter GH, Schechterly C, Lebedeva M, Operskalski E, Luban NL, Alter H, Busch MP, Slichter SJ, Nomis PJ. Leukoreduction and ultraviolet treatment reduce both the magnitude and the duration of the HLA antibody response. Transfusion 2014, 54, 672-680.

18. Jagodich TA, Holowaychuk MK. Transfusion practice in dogs and cats: an internet-based survey. J Vet Emerg Crit Care (San Antonio) 2016, 26, 360-372.

19. Janatpour K, Denning L, Nelson K, Betlach B, Mackenzie M, Holland P. Comparison of X-ray vs. gamma irradiation of CPDA-1 red cells. Vox Sang 2005, 89, 215-219.

20. Jy W, Horstman LL, Ahn YS. Microparticle size and its relation to composition, functional activity, and clinical significance. Semin Thromb Hemost 2010, 36, 876-880.

21. Kisielewicz C, Self IA. Canine and feline blood transfusions: controversies and recent advances in administration practices. Vet Anaesth Analg 2014, 41, 233-242.

22. Kumar P, Thapliyal R, Coshic P, Chatterjee K. Retrospective evaluation of adverse transfusion reactions following blood product transfusion from a tertiary care hospital: a preliminary step towards hemovigilance. Asian J Transfus Sci 2013, 7, 109-115.

23. Maglaras CH, Koenig A, Bedard DL, Brainard BM. Retrospective evaluation of the effect of red blood cell product age on occurrence of acute transfusion-related complications in dogs: 210 cases (2010-2012). J Vet Emerg 
Crit Care (San Antonio) 2017, 27, 108-120.

24. McMichael MA, Smith SA, Galligan A, Swanson KS, Fan TM. Effect of leukoreduction on transfusion-induced inflammation in dogs. J Vet Intern Med 2010, 24, 1131-1137.

25. Phelan HA, Eastman AL, Aldy K, Carroll EA, Nakonezny PA, Jan T, Howard JL, Chen Y, Friese RS, Minei JP. Prestorage leukoreduction abrogates the detrimental effect of aging on packed red cells transfused after trauma: a prospective cohort study. Am J Surg 2012, 203, 198-204.

26. Phelan HA, Gonzalez RP, Patel HD, Caudill JB, Traylor RK, Yancey LR, Sperry JL, Friese RS, Nakonezny PA. Prestorage leukoreduction ameliorates the effects of aging on banked blood. J Trauma 2010, 69, 330-337.

27. Purcell SL, Claus M, Hosgood G, Smart L. Effect of leukoreduction on concentrations of interleukin-8, interleukin$1 \beta$, and tumor necrosis factor- $\alpha$ in canine packed red blood cells during storage. Am J Vet Res 2015, 76, 969-974.

28. Rajesh K, Harsh S, Amarjit K. Effects of prestorage leukoreduction on the rate of febrile nonhemolytic transfusion reactions to red blood cells in a tertiary care hospital. Ann Med Health Sci Res 2015, 5, 185-188.

29. Shanwell A, Kristiansson M, Remberger M, Ringdén O. Generation of cytokines in red cell concentrates during storage is prevented by prestorage white cell reduction. Transfusion 1997, 37, 678-684.

30. Smith HM, Farrow SJ, Ackerman JD, Stubbs JR, Sprung J. Cardiac arrests associated with hyperkalemia during red blood cell transfusion: a case series. Anesth Analg 2008, 106, 1062-1069.

31. Treleaven J, Gennery A, Marsh J, Norfolk D, Page L, Parker
A, Saran F, Thurston J, Webb D. Guidelines on the use of irradiated blood components prepared by the British Committee for Standards in Haematology blood transfusion task force. Br J Haematol 2011, 152, 35-51.

32. von Fliedner V, Higby DJ, Kim U. Graft-versus-host reaction following blood product transfusion. Am J Med 1982, 72, 951-961.

33. Wadhwa M, Seghatchian MJ, Dilger P, Sands D, Krailadisiri P, Contreras M, Thorpe R. Cytokines in WBC-reduced apheresis PCs during storage: a comparison of two WBCreduction methods. Transfusion 2000, 40, 1118-1126.

34. Weiskopf RB, Schnapp S, Rouine-Rapp K, Bostrom A, Toy P. Extracellular potassium concentrations in red blood cell suspensions after irradiation and washing. Transfusion 2005, 45, 1295-1301.

35. Williamson LM, Stainsby D, Jones H, Love E, Chapman CE, Navarrete C, Lucas G, Beatty C, Casbard A, Cohen H. The impact of universal leukodepletion of the blood supply on hemovigilance reports of posttransfusion purpura and transfusion-associated graft-versus-host disease. Transfusion 2007, 47, 1455-1467.

36. Yu DH, Nho DH, Song RH, Kim SH, Lee MJ, Nemzek JA, Park J. High-mobility group box 1 as a surrogate prognostic marker in dogs with systemic inflammatory response syndrome. J Vet Emerg Crit Care (San Antonio) 2010, 20, 298-302.

37. Zimring JC, Spitalnik SL. To RBC or not to RBC: the role of suicidal death in hemolytic transfusion reactions. Transfusion 2009, 49, 1776-1778. 